\title{
NOTE ON INTERCHANGE OF ORDER OF LIMITS*
}

\author{
BY T. H. HILDEBRANDT
}

There exist a number of theorems giving necessary and sufficient conditions for the relation

$$
\lim _{m} \lim _{n} a_{m n}=\lim _{n} \lim _{m} a_{m n},
$$

$\left\{a_{m n}\right\}$ being a double sequence of real numbers. Most of these theorems are not symmetric in $m$ and $n$, which is only natural, because as a rule wherever the interchange of order of limits is in question, there is information about the iterated limit in one order, and the existence of the limit in reverse order is desired. Nevertheless it may be of interest to deduce a condition which is symmetric in $m$ and $n$.

By way of notation, we shall assume that the symbol $\lim _{m} \lim _{n} a_{m n}$ implies that for every $m, \lim _{n} a_{m n}$ exists. On the other hand, we shall use the symbol $\lim _{m}{\underline{\lim _{n}}}_{m} a_{m n}$ with the implication that

$$
\lim _{m} \varlimsup_{\lim _{n}} a_{m n}=\lim _{m} \underline{\lim }_{n} a_{m n} .
$$

Then, obviously, if we deduce necessary and sufficient conditions for the relation

$$
\lim _{m}{\underline{\varlimsup_{n}}}_{n} a_{m n}=\lim _{n}{\varlimsup_{m}}_{m} a_{m n},
$$

those for the equality mentioned at the outset require in addition the assumption of the existence of $\lim _{n} a_{m n}$ for every $m$ and of $\lim _{m} a_{m n}$ for every $n$.

Let us deduce necessary conditions. Let $\varlimsup_{m} a_{m n}=b_{n}$ and $\lim _{m} a_{m n}=c_{n}$, and $\lim _{n} b_{n}=\lim _{n} c_{n}=d$. Then for every $\epsilon>0$, there exists an $n_{\epsilon}$ such that when $n \geqq n_{\epsilon}$, we have

$$
\left|b_{n}-d\right| \leqq \epsilon \text { and }\left|c_{n}-d\right| \leqq \epsilon .
$$

From the definition of $b_{n}$ and $c_{n}$ we have, for every $n$, and

\footnotetext{
* Presented to the Society, September 8, 1927.
} 
every $\epsilon>0$, an $m_{\epsilon n}$ (depending on $\epsilon$ and $n$ ) such that when $m \geqq m_{\epsilon n}$, we have

$$
c_{n}-\epsilon \leqq a_{m n} \leqq b_{n}+\epsilon .
$$

By combining these two statements, we obtain the following. For every $\epsilon>0$, there exists an $n_{\epsilon}$ such that for every $n_{1} \geqq n_{\epsilon}$, there exists an $m_{\epsilon n_{1}}$ such that for every $m_{2} \geqq m_{\epsilon n_{1}}$, we have

$$
d-2 \epsilon \leqq a_{m_{2} n_{1}} \leqq d+2 \epsilon,
$$

i. e.,

$$
\left|a_{m_{2} n_{1}}-d\right| \leqq 2 \epsilon \text {. }
$$

For future reference we shall call this Statement A. Similarly, from the fact that $\lim _{m} \overline{\lim }_{n} a_{m n}=d$, we have: For every $\epsilon>0$ there exists an $m_{\epsilon}$ such that for every $m_{1} \geqq m_{\epsilon}$, there exists an $n_{\epsilon m_{1}}$ such that for every $n_{2} \geqq n_{\epsilon m_{1}}$, we have

$$
\left|a_{m_{1} n_{2}}-d\right| \leqq 2 \epsilon \text {. }
$$

By combining these two statements into one, and replacing $4 \epsilon$ by $\epsilon$, we get the necessary condition desired, viz.: For every $\epsilon>0$, there exists an $n_{\epsilon}$ and an $m_{\epsilon}$ such that for every $n_{1} \geqq n_{\epsilon}$ and $m_{1} \geqq m_{\epsilon}$, there exists an $m_{\epsilon n_{1}}$ and an $n_{\epsilon m_{1}}$ such that for every $m_{2} \geqq m_{\epsilon n_{1}}$ and $n_{2} \geqq n_{\epsilon m_{1}}$, we have

$$
\left|a_{m_{1} n_{2}}-a_{m_{2} n_{1}}\right| \leqq \epsilon \text {. }
$$

This condition is also sufficient. For suppose the condition satisfied. Then for a particular $m_{1}$ and $n_{2}$ chosen in accordance with the specifications, and for every $n_{1} \geqq n_{\epsilon}$ and every $m_{2} \geqq m_{\epsilon n_{1}}$, we have

$$
a_{m_{1} n_{2}}-\epsilon \leqq a_{m_{2} n_{1}} \leqq a_{m_{1} n_{2}}+\epsilon .
$$

From this we conclude that for every $n_{1} \geqq n_{\epsilon}$, the greatest and the least of the limits $b_{n}$ and $c_{n}$ satisfy the conditions

$$
a_{m_{1} n_{2}}-\epsilon \leqq c_{n_{1}} \leqq b_{n_{1}} \leqq a_{m_{1} n_{2}}+\epsilon .
$$

This has as consequence that if $n_{1}$ and $n_{0}$ are greater than or equal to $n_{\epsilon}$, then

$$
\left|b_{n_{0}}-b_{n_{1}}\right| \leqq 2 \epsilon,\left|c_{n_{0}}-c_{n_{1}}\right| \leqq 2 \epsilon \text {, and }\left|c_{n_{1}}-b_{n_{1}}\right| \leqq 2 \epsilon .
$$


Hence $\lim _{n} c_{n}$ and $\lim _{n} b_{n}$ exist and are equal, i.e. $\lim _{n} \overline{\lim }_{m} a_{m n}$ exists. From the symmetry of the condition, we conclude that $\lim _{m}{\lim _{n}}_{n n}$ exists also. The identity of the two limits is then a consequence of the condition of our theorem and Statement A.

We note finally that the Cauchy condition for convergence of the double limit, $\lim _{m n} a_{m n}$, is the special case of our condition in which $m_{\epsilon n_{1}}$ and $n_{\epsilon m_{1}}$ are independent of $n_{1}$ and $m_{1}$ respectively, and can therefore be taken as $m_{e}$ and $n_{\epsilon}$, respectively.

UNIVERSITY OF MICHIGAN

\title{
ON BOUNDED REGULAR FRONTIERS IN THE PLANE*
}

\author{
BY W. A. WILSON
}

1. Introduction. The term regular frontier has been introduced by $\mathrm{P}$. Urysohn $\uparrow$ to designate a continuum which is the frontier of two or more components of its complement. Regular frontiers in the plane have been discussed by various authors. A. Rosenthal $\ddagger$ has shown that a continuum which is the union of two bounded continua that are irreducible between the same pair of points and have no other common points is a regular frontier. R. L. Moore§ has given necessary and sufficient conditions that a bounded continuum be a regular frontier whose complement has exactly two components. C. Kuratowski\| has given necessary conditions for a continuum to be a regular frontier which is the frontier of every component of its complement.

* Presented to the Society, October 29, 1927.

$\dagger \mathrm{P}$. Urysohn, Mémoire sur les multiplicités Cantoriennes, Fundamenta Mathematicae, vol. 7, p. 98.

$\ddagger$ A. Rosenthal, Teilung der Ebene durch Irreduzible Kontinua, Sitzungsberichte der Münchener Akademie, 1919.

$\S$ R. L. Moore, Concerning the common boundary of two domains, Fundamenta Mathematicae, vol. 6, pp. 203-213.

\| C. Kuratowski, Sur les coupures du plan, Fundamenta Mathematicae, vol. 6 , pp. 130-145. 